\title{
Antibacterial Activity of the Ethanol Extract of Paeonia Lactiflora on Growth of Oral Bacteria
}

\author{
Kyung-Yeol Lee ${ }^{1}$, Jeong-Dan Cha ${ }^{1}$, Sung-Mi Choi ${ }^{2}$, Eun-Jin Jang ${ }^{3}$, Eun-Sil Ko ${ }^{1}$, Su-Mi Cha ${ }^{1}$ and \\ Soon-Il Yun ${ }^{4}$ \\ ${ }^{1}$ Department of Oral Microbiology and Institute of Oral Bioscience, Chonbuk National University, Jeonju, Republic of Korea \\ ${ }^{2}$ Department of Dental Hygiene, Daegu Health College, Daegu, Republic of Korea \\ ${ }^{3}$ Department of Dental Technology, Daegu Health College, Daegu, Republic of Korea \\ ${ }^{4}$ Department of Food Science \& Technology, College of Agriculture \& Life Sciences, Chonbuk National University, Jeonju, \\ Republic of Korea
}

*Corresponding author: Prof. Soon-Il Yun, Department of Food Science \& Technology, College of Agriculture \& Life Sciences, Chonbuk National University, 664-14 Duckjin-Dong, Duckjin-Ku, Jeonju, Chonbuk, 561-756 Republic of Korea, Fax: +82-63-270-4049, Tel: +82-63-270-2566, E-mail: siyun@jbnu.ac.kr

Citation: Kyung-Yeol Lee, Jeong-Dan Cha, Sung-Mi Choi, Eun-Jin Jang, Eun-Sil Ko, et al. (2018) Antibacterial Activity of the Ethanol Extract of Paeonia Lactiflora on Growth of Oral Bacteria. J Oral Health Dent 2: 201

Article history: Received: 28 December 2017, Accepted: 26 February 2018, Published: 28 February 2018

\begin{abstract}
The roots of Paeonia lactiflora Pallas are important crude drugs in Korean traditional medicine. A decoction of the dried root has been used in the treatment of rheumatoid arthritis, systemic lupus erythematosus, dysmenorrhea, hepatitis, muscle cramping, inflammation, spasms, and fever and stimulate blood circulation. In this study, the combination effect of the ethanol extract of Paeonia lactiflora (PLE) was evaluated against oral bacteria, either alone or with antibiotics, via broth dilution method and checkerboard and time kill assay. In these results, MIC/MBC values for PLE against all the tested bacteria ranged between 250-2000/250-2000 microg/mL, for ampicillin 0.031-16/0.125-32 microg/mL, gentamicin 2-256/4-512 microg/mL, erythromycin 0.008-32/0.016-64 microg/mL, and vancomycin 0.564/1-128 microg/mL, respectively. Furthermore, the MIC and MBC were reduced to one half-four as a result of the combination of PLE with antibiotics. Six hours of treatment with 1/2 MIC of PLE with 1/2 MIC of antibiotics resulted from an increase of the rate of killing in units of $\mathrm{CFU} / \mathrm{mL}$ to a greater degree than was observed with alone. These results suggest that the ethanol extract of Paeonia lactiflora (PLE) is important in the antibacterial actions of oral pathogen agents.
\end{abstract}

Keywords: Paeonia lactiflora Pallas; Antibacterial activity; Oral pathogen bacteria; Synergistic effect; Minimum inhibitory concentrations (MICs); Minimum bactericidal concentrations (MBCs)

List of abbreviations: PLE: the ethanol extract of Paeonia lactiflora Pallas; MICs: Minimum inhibitory concentrations; MBCs: Minimum bactericidal concentrations; CFU: Colony Forming Unit; FIC index: Fractional Inhibitory Concentration; FBC index: Fractional Bactericidal Concentration index

\section{Introduction}

More than 700 different bacterial species have been detected in the oral cavity of humans [1]. Saliva contains $10^{8}$ to $10^{9}$ bacteria per milliliter, and some of these adhere to the teeth and initiate formation of a dental biofilm, previously called dental plaque [1]. Oral diseases are major health problems with dental caries and periodontal diseases among the most important preventable global infectious diseases [2]. Oral health influences the general quality of life and poor oral health is linked to chronic conditions and systemic diseases [3]. The development of dental caries involves acidogenic and aciduric gram-positive bacteria, primarily the mutans streptococci (Streptococcus mutans and S. sobrinus), lactobacilli, and actinomycetes, which metabolize sucrose to organic acids (mainly lactic acid) that dissolve the calcium phosphate in teeth, causing decalcification and eventual decay [2,4].

Periodontal disease results from chronic infection and inflammation of the tissues that support the teeth [2,5]. This oral inflammatory disease is driven by pathogenic succession characterized by a shift in microbial species in the gingival sulcus from gram-positive, facultative and fermentative microorganisms to predominantly gram-negative, anaerobic, chemo-organotrophic, and proteolytic microorganisms [5,6]. Periodontal disease also is implicated in several systemic diseases, including preterm/low birth weight deliveries, cardiovascular events, diabetes and other systemic conditions [5-7]. 
Several antibacterial agents including, fluorides, phenol derivatives, ampicillin, erythromycin, penicillin, tetracycline, and vancomycin have been used widely in dentistry to inhibit bacterial growth [8,9]. However, excessive use of these chemicals can result in derangements of the oral and intestinal flora and cause side effects such as microorganism susceptibility, vomiting, diarrhea and tooth staining [10]. These problems necessitate further search for natural antibacterial agents that are safe for humans and specific for oral pathogens. Natural products have recently been investigated more thoroughly as promising agents to prevent oral diseases, especially plaque-related diseases such as dental caries $[11,12]$.

Paeonia lactiflora Pallas belongs to the genus Paeonia in the family Paeoniaceae, which is an important crude drug in Korean traditional medicine [13]. A decoction of the dried root without bark has been used in the treatment of rheumatoid arthritis, systemic lupus erythematosus, hepatitis, dysmenorrheal, muscle cramping, inflammation, and spasms, and fever for centuries [13-15]. Monoterpene glycosides, such as albiflorin, oxypaeoniflorin, and paeoniflorin, have been isolated from P. lactiflora roots with galloyl and phenolic compounds, including benzoic acid, catechin, gallic acid, methyl gallate, paeonol, and 1,2,3,4,6-penta$O$-galloyl- $\beta$-D-glucose (PGG) [16]. These compounds have been revealed to have anti-inflammatory, anti-diabetic, anti-oxidant, anti-allergic, anti-coagulative, sedative, analgesic acitivities, and etc. Paeoniflorin, a major constituent of Paeoniae Radix, has been reported to exhibit diverse biological activities, including anti-inflammatory, spasmolytic, immune-regulating, and gastroprotective activities $[17,18]$.

Phytotherapy has many potentially significant advantages associated with the synergistic interactions like, increased efficiency, reduction of undesirable effects, increase in the stability or bioavailability of the free agents and obtaining an adequate therapeutic effect with relatively small doses, when compared with a synthetic medication [19]. Recently, plant antimicrobials have been found to be synergistic enhancers in that though they may not have any antimicrobial properties alone, but when they are taken concurrently with standard drugs they enhance the effect of that drug [20]. Although, plant derived antimicrobial are less potent, this enhances the need to research a synergism interaction between plant bioactive products and antimicrobial agent. Therefore, the association between plant extracts and synthetic drugs has shed light to a novel approach in controlling multidrug resistant strains and in modulating the action of antibiotics $[19,20]$.

The aim of the present study was to examine antibacterial effect of Paeonia lactiflora extract (PLE) against oral pathogens and to investigate the synergy between PLE and commonly used antibiotics.

\section{Material and Methods}

\section{Plant material and preparation of 50\% ethanol extract of Paeonia lactiflora extract (PLE)}

Dried flowers from P. lactiflora (PLE, $2 \mathrm{~kg}$ ) were macerated and extracted three times with 50\% EtOH (10 L) for $4 \mathrm{~h}$ at $80{ }^{\circ} \mathrm{C}$. The combined $50 \% \mathrm{EtOH}$ extract $(30 \mathrm{~L})$ was clarified by filtration and evaporated to obtain brown syrup (300 g). One hundred mg/ $\mathrm{mL}$ of extract was dissolved in 10\% dimethyl sulfoxide (DMSO, Sigma Chemical Co., St. Louis, MO, USA) and then, diluted with bacteria culture medium for testing. All of the extract was kept at $4{ }^{\circ} \mathrm{C}$ in the dark until further use.

\section{Bacterial strains}

The oral bacterial strains used in this study were: cariogenic bacterial strains, Streptococcus mutans ATCC 25175 (American Type Culture Collection), Streptococcus sanguinis ATCC 10556, Streptococcus sobrinus ATCC 27607, Streptococcus ratti KCTC (Korean Collection for Type Cultures) 3294, Streptococcus criceti KCTC 3292, Streptococcus parasanguinis KCOM 1497 (Korean Collection for Oral Microbiology), Streptococcus downei KCOM 1165, Streptococcus anginosus ATCC 31412, and Streptococcus gordonii ATCC 10558 and periodontopathogenic bacterial strains, Actinobacillus actinomycetemcomitans ATCC 43717, Fusobacterium nucleatum ATCC 10953, and Porphylomonas gingivalis ATCC 33277. Brain-Heart Infusion (BHI) broth supplemented with 1\% yeast extract (Difco Laboratories, Detroit, MI) was used for cariogenic bacterial strains (facultative anaerobic bacteria). For periodontopathogenic bacterial strains (microaerophilic and obligate anaerobic bacteria), BHI broth containing hemin $1 \mu \mathrm{g} / \mathrm{mL}$ (Sigma, St. Louis, MO, USA) and menadione $1 \mu \mathrm{g} / \mathrm{mL}$ (Sigma) was used.

\section{Minimum inhibitory concentrations/minimum bactericidal concentrations assay}

The minimum inhibitory concentrations (MICs) were determined for PLE by the broth dilution method, and were carried out in triplicate. The antibacterial activities were examined after incubation at $37{ }^{\circ} \mathrm{C}$ for $18 \mathrm{~h}$ (facultative anaerobic bacteria), for $24 \mathrm{~h}$ (microaerophilic bacteria), and for 1-2 days (obligate anaerobic bacteria) under anaerobic conditions. The inoculums suspension containing $0.125-4 \mathrm{mg} / \mathrm{mL}$ PLE was added to give a final concentration of between $5 \times 10^{6}$ and $8 \times 10^{6} \mathrm{CFU} / \mathrm{mL}$ for the assays. MICs were determined as the lowest concentration of test samples that resulted in a complete inhibition of visible growth in the broth. $\mathrm{MIC}_{50} \mathrm{~s}$ and $\mathrm{MIC}_{90} \mathrm{~s}$, defined as MICs at which, 50 and $90 \%$, respectively of oral bacteria were inhibited, were determined. Following anaerobic incubation of MICs plates, the minimum bactericidal concentrations (MBCs) were determined on the basis of the lowest concentration of PLE that kills $99.9 \%$ of the test bacteria by plating out onto each appropriate agar plate. Ampicillin, gentamicin, erythromycin, and vancomycin (Sigma) were used as standard antibiotics in order to compare the sensitivity of PLE against oral bacteria. 


\section{Checker-board dilution test}

The antibacterial effects of a combination of PLE, which exhibited the highest antimicrobial activity and antibiotics, were assessed by the checkerboard test as previously described [21]. The antimicrobial combinations assayed included PLE with ampicillin, gentamicin, erythromycin, and/or vancomycin. Serial dilutions of two different antimicrobial agents were mixed in cation-supplemented Mueller-Hinton broth. After $24-48 \mathrm{~h}$ of incubation at $37^{\circ} \mathrm{C}$, the MICs were determined to be the minimal concentration at which there was no visible growth and MBCs were determined on the basis of the lowest concentration of PLE that kills $99.9 \%$ of the test bacteria by plating out onto each appropriate agar plate. The fractional inhibitory concentration (FIC)/ fractional bactericidal concentration $(\mathrm{FBC})$ index was calculated according to the equation: $\mathrm{FIC} / \mathrm{FBC}$ index $=\mathrm{FIC} / \mathrm{FBC}+\mathrm{FIC} /$ $\mathrm{FBC}_{\mathrm{B}}=(\mathrm{MIC} / \mathrm{MBC}$ of drug $\mathrm{A}$ in combination/MIC/MBC of drug $\mathrm{A}$ alone $)+(\mathrm{MIC} / \mathrm{MBC}$ of drug $\mathrm{B}$ in combination/MIC/MBC of drug B alone). The FIC and FBC index are the sum of the FICs and FBCs of each of the drugs, which in turn is defined as the MIC and MBC of each drug when it is used in combination divided by the MIC and MBC of the drug when it is used alone. The interaction was defined as synergistic if the FIC and FBC index was less than or equal to 0.5, additive if the FIC and FBC index was greater than 0.5 and less than or equal 1.0, indifferent if the FIC and FBC index was greater than 1.0 and less than or equal to 2.0, and antagonistic if the FIC and FBC index was greater than 2.0 [21].

\section{Time-kill curves}

Bactericidal activities of the drugs under study were also evaluated using time-kill curves on oral bacteria. Tubes containing Mueller-Hinton supplemented to which antibiotics had been added at concentrations of the $1 / 2$ MIC were inoculated with a suspension of the test strain, giving a final bacterial count between $5 \sim 8 \times 10^{6} \mathrm{CFU} / \mathrm{mL}$. The tubes were thereafter incubated at $37^{\circ} \mathrm{C}$ in an anaerobic chamber and viable counts were performed at $0,0.5,1,2,3,6,9,12,18$ and $24 \mathrm{~h}$ after addition of antimicrobial agents, on agar plates incubated for up to $48 \mathrm{~h}$ in anaerobic chamber at $37^{\circ} \mathrm{C}$. Antibiotic carryover was minimized by washings by centrifugation and serial 10-fold dilution in sterile phosphate-buffered saline, $\mathrm{pH}$ 7.3. Colony counts were performed in duplicate, and means were taken. The solid media used for colony counts were BHI agar for streptococci and BHI agar containing hemin and menadione for P. intermedia and P. gingivalis.

\section{Results and Discussion}

The PLE was evaluated for their antimicrobial activities against twelve common bacterial species present in the oral cavity. The results of the antimicrobial activity showed that PLE exhibited antimicrobial activities against cariogenic bacteria (MICs, 250 to $1000 \mu \mathrm{g} / \mathrm{mL}$; MBCs, 250 to $2000 \mu \mathrm{g} / \mathrm{mL}$ ), against periodontopathogenic bacteria (MICs, 250 to $2000 \mu \mathrm{g} / \mathrm{mL}$; MBCs, 500 to 2000 $\mu \mathrm{g} / \mathrm{mL}$ ) and for ampicillin, either $0.31 / 0.125$ or $16 / 32 \mu \mathrm{g} / \mathrm{mL}$; for gentamicin, either $2 / 4$ or $256 / 512 \mu \mathrm{g} / \mathrm{mL}$; for erythromycin, either $0.008 / 0.016$ or $32 / 64 \mu \mathrm{g} / \mathrm{mL}$; for vancomycin, either $0.5 / 1$ or $64 / 128 \mu \mathrm{g} / \mathrm{mL}$ on tested all bacteria (Table 1 ). The range of MIC ${ }_{50}$ PLE was from 11 to $953 \mu \mathrm{g} / \mathrm{mL}$. The PLE showed stronger antimicrobial activity against S. gordonii than other bacteria.

\begin{tabular}{|c|c|c|c|c|c|c|c|}
\hline \multirow{3}{*}{$\begin{array}{c}\text { Samples } \\
\text { S. mutans } \\
\text { ATCC } 25175^{1}\end{array}$} & \multicolumn{3}{|c|}{ PLE } & Ampicillin & Gentamicin & Erythromycin & Vancomycin \\
\hline & $\mathrm{MIC}_{50<}$ & $\mathrm{MIC}_{90<}$ & $\begin{array}{c}\mathrm{MIC} / \mathrm{MBC} \\
(\mu \mathrm{g} / \mathrm{mL})\end{array}$ & \multicolumn{4}{|c|}{$\begin{array}{c}\mathrm{MIC} / \mathrm{MBC} \\
(\mu \mathrm{g} / \mathrm{mL})\end{array}$} \\
\hline & 63 & 250 & $250 / 250$ & $0.25 / 0.25$ & $8 / 16$ & $0.063 / 0.125$ & $1 / 2$ \\
\hline $\begin{array}{l}\text { S. sanguinis } \\
\text { ATCC } 10556\end{array}$ & 76 & 500 & $500 / 1000$ & $0.125 / 0.5$ & $8 / 32$ & $0.016 / 0.031$ & $0.5 / 1$ \\
\hline $\begin{array}{l}\text { S. parasanguinis } \\
\text { KCOM } 1497^{2}\end{array}$ & 46 & 1000 & $1000 / 1000$ & $0.5 / 1$ & $16 / 32$ & $0.25 / 0.5$ & $2 / 4$ \\
\hline $\begin{array}{l}\text { S. sobrinus } \\
\text { ATCC } 27607\end{array}$ & 132 & 500 & $500 / 1000$ & $0.063 / 0.125$ & $8 / 32$ & $0.031 / 0.063$ & $1 / 2$ \\
\hline $\begin{array}{c}\text { S. ratti } \\
\text { KCTC } 3294^{3}\end{array}$ & 187 & 500 & $500 / 1000$ & $0.25 / 0.5$ & $8 / 16$ & $0.008 / 0.016$ & $1 / 1$ \\
\hline $\begin{array}{c}\text { S. criceti } \\
\text { KCTC } 3292\end{array}$ & 184 & 500 & $500 / 2000$ & $0.031 / 0.125$ & $16 / 32$ & $0.125 / 0.25$ & $2 / 4$ \\
\hline $\begin{array}{c}\text { S. downei } \\
\text { KCOM } 1165\end{array}$ & 29 & 1000 & $1000 / 1000$ & $2 / 4$ & $32 / 64$ & $0.25 / 0.5$ & $8 / 16$ \\
\hline $\begin{array}{l}\text { S. anginosus } \\
\text { ATCC } 31412\end{array}$ & 32 & 1000 & $1000 / 2000$ & $0.063 / 0.25$ & $8 / 16$ & $0.25 / 0.5$ & $1 / 4$ \\
\hline $\begin{array}{l}\text { S. gordonii } \\
\text { ATCC } 10558\end{array}$ & 11 & 250 & $250 / 250$ & $0.125 / 0.25$ & $16 / 32$ & $0.031 / 0.063$ & $0.5 / 1$ \\
\hline $\begin{array}{c}\text { A. actinomycetemcomitans } \\
\text { ATCC } 43717\end{array}$ & 95 & 2000 & $2000 / 2000$ & $16 / 32$ & $16 / 16$ & $0.125 / 0.25$ & $2 / 4$ \\
\hline $\begin{array}{l}\text { F. nucleatum } \\
\text { ATCC } 51190\end{array}$ & 82 & 500 & $500 / 1000$ & $4 / 16$ & $2 / 4$ & $32 / 64$ & $64 / 128$ \\
\hline
\end{tabular}




\begin{tabular}{|c|c|c|c|c|c|c|c|}
\hline \multirow{2}{*}{ Samples } & \multicolumn{3}{|c|}{ PLE } & Ampicillin & Gentamicin & Erythromycin & Vancomycin \\
\cline { 2 - 7 } & MIC $_{50<}$ & MIC $_{\mathbf{9 0 <}}$ & $\begin{array}{c}\text { MIC/MBC } \\
(\mu \mathrm{g} / \mathrm{mL})\end{array}$ & \multicolumn{2}{|c|}{$\begin{array}{c}\text { MIC/MBC } \\
(\mu \mathrm{g} / \mathrm{mL})\end{array}$} \\
\hline $\begin{array}{c}\text { P. intermedia } \\
\text { ATCC 49049 }\end{array}$ & 953 & 1000 & $1000 / 2000$ & $1 / 2$ & $32 / 64$ & $16 / 32$ & $16 / 36$ \\
\hline $\begin{array}{c}\text { P. gingivalis } \\
\text { ATCC 33277 }\end{array}$ & 124 & 250 & $250 / 500$ & $0.25 / 0.5$ & $256 / 512$ & $2 / 8$ & $16 / 16$ \\
\hline
\end{tabular}

${ }^{1}$ American Type Culture Collection (ATCC)

${ }^{2}$ Korean collection for Oral Microbiology (KCOM)

${ }^{3}$ Korean collection for Type Cultures (KCTC)

Table 1: Antibacterial activity of 50\% ethanol extract of Paeonia lactiflora pallas (PLE) and antibiotics in oral bacteria

Natural products are a major source of chemical diversity and have provided important therapeutic agents for many bacterial diseases $[12,13,19,20]$. Combinations of some herbal materials and different antibiotics might affect the inhibitory effect of these antibiotics [20,21]. It is always suggested to treat bacterial infections with a combination of antimicrobial agents for the prevention of drug resistance development and to improve efficacy. Drug combinations having synergistic interactions are generally considered as more effective and, therefore, preferable [19,20].

The synergistic effects of the PLE alone or with antibiotics were evaluated in oral bacteria (Table 2,3,4 and 5). In combination with MICs for ampicillin, PLE was reduced $\geq 4$-fold in cariogenic bacteria, producing a synergistic effect as defined by FICI $\leq 0.5$ except S. sobrinus and $S$. anginosus, and in all tested periodontopathogenic bacteria by FICI $\leq 0.5$. The MBC for ampicillin was shown synergistic effects in $S$. sanguinis, $S$. ratti, and $S$. criceti by FBCI $\leq 0.5$, and in the periodontopathogenic bacteria, $P$. intermedia by FBCI $\leq 0.5$ (Table 2). In combination with MIC of gentamicin, the PLE was reduced $\geq 4-8$-fold in all tested bacteria expect $S$. mutans and $S$. downei by FICI $\geq 0.75$ and MBC in S. mutans, S. sanguinis, S. ratti, S.downei, S. gordonii, and A. actinomycetemcomitans by FBCI $\geq 0.75$ (Table 3). In combination with MIC of erythromycin, the PLE was reduced $\geq 4$-8-fold in all tested bacteria expect $S$. sanguinis, S. sobrinus, and S. gingivalis by FICI $\geq 0.75$ and MBC in S. sanguinis, S. sobrinus, and S. gordonii by FBCI $\geq 0.75$ (Table 4 ). In combination with MICs for vancomycin, PLE was reduced $\geq 4$-fold in all tested bacteria, producing a synergistic effect as defined by FICI $\leq 0.5$ except $S$. parasanguinis, $S$. sobrinus, and $S$. criceti by FICI $\geq 0.75$, and MBC for vancomycin was shown synergistic effects in all tested bacteria except S. sanguinis, S. parasanguinis, S. gordonii, and A. actinomycetemcomitans by FICI $\geq 0.75$ (Table $5)$.

\begin{tabular}{|c|c|c|c|c|c|c|}
\hline \multirow{2}{*}{ Strains } & \multirow{2}{*}{ Agent } & \multicolumn{2}{|c|}{$\mathrm{MIC} / \mathrm{MBC}(\mu \mathrm{g} / \mathrm{mL})$} & \multirow{2}{*}{ FIC/FBC } & \multirow{2}{*}{$\begin{array}{l}\text { FICI/ } \\
\text { FBCI }^{2}\end{array}$} & \multirow{2}{*}{ Outcome } \\
\hline & & Alone & Combination $^{1}$ & & & \\
\hline \multirow{2}{*}{$\begin{array}{l}\text { S. mutans } \\
\text { ATCC } 25175^{3}\end{array}$} & PLE & $250 / 250$ & $63 / 125$ & $0.25 / 0.5$ & \multirow{2}{*}{$0.5 / 1.0$} & \multirow{2}{*}{$\begin{array}{l}\text { Synergistic/ } \\
\text { Additive }\end{array}$} \\
\hline & Ampicillin & $0.25 / 0.25$ & $0.063 / 0.125$ & $0.25 / 0.5$ & & \\
\hline \multirow{2}{*}{$\begin{array}{l}\text { S. sanguinis } \\
\text { ATCC } 10556\end{array}$} & PLE & $500 / 1000$ & $125 / 250$ & $0.25 / 0.25$ & \multirow{2}{*}{$0.5 / 0.5$} & \multirow{2}{*}{$\begin{array}{l}\text { Synergistic/ } \\
\text { Synergistic }\end{array}$} \\
\hline & Ampicillin & $0.125 / 0.5$ & $0.031 / 0.125$ & $0.25 / 0.25$ & & \\
\hline \multirow{2}{*}{$\begin{array}{l}\text { S. parasanguinis } \\
\text { KCOM } 1497^{4}\end{array}$} & PLE & $1000 / 1000$ & $250 / 500$ & $0.25 / 0.5$ & \multirow{2}{*}{$0.5 / 0.75$} & \multirow{2}{*}{$\begin{array}{l}\text { Synergistic/ } \\
\text { Additive }\end{array}$} \\
\hline & Ampicillin & $0.5 / 1$ & $0.125 / 0.25$ & $0.25 / 0.25$ & & \\
\hline \multirow{2}{*}{$\begin{array}{l}\text { S. sobrinus } \\
\text { ATCC } 27607\end{array}$} & PLE & $500 / 1000$ & $250 / 500$ & $0.5 / 0.5$ & \multirow{2}{*}{$0.75 / 0.75$} & \multirow{2}{*}{$\begin{array}{l}\text { Synergistic/ } \\
\text { Additive }\end{array}$} \\
\hline & Ampicillin & $0.063 / 0.125$ & $0.016 / 0.031$ & $0.25 / 0.25$ & & \\
\hline \multirow{2}{*}{$\begin{array}{c}\text { S. ratti } \\
\text { KCTC } 3294^{5}\end{array}$} & PLE & $500 / 1000$ & $125 / 250$ & $0.25 / 0.25$ & \multirow{2}{*}{$0.5 / 0.5$} & \multirow{2}{*}{$\begin{array}{l}\text { Synergistic/ } \\
\text { Synergistic }\end{array}$} \\
\hline & Ampicillin & $0.25 / 0.5$ & $0.063 / 0.125$ & $0.25 / 0.25$ & & \\
\hline \multirow{2}{*}{$\begin{array}{c}\text { S. criceti } \\
\text { KCTC } 3292\end{array}$} & PLE & $500 / 2000$ & $125 / 500$ & $0.25 / 0.25$ & \multirow{2}{*}{$0.5 / 0.5$} & \multirow{2}{*}{$\begin{array}{l}\text { Synergistic/ } \\
\text { Synergistic }\end{array}$} \\
\hline & Ampicillin & $0.031 / 0.125$ & $0.008 / 0.031$ & $0.25 / 0.25$ & & \\
\hline \multirow{2}{*}{$\begin{array}{c}\text { S. downei } \\
\text { KCOM } 1165\end{array}$} & PLE & $1000 / 1000$ & $250 / 500$ & $0.25 / 0.5$ & \multirow{2}{*}{$0.5 / 0.75$} & \multirow{2}{*}{$\begin{array}{l}\text { Synergistic/ } \\
\text { Additive }\end{array}$} \\
\hline & Ampicillin & $2 / 4$ & $0.5 / 1$ & $0.25 / 0.25$ & & \\
\hline \multirow{2}{*}{$\begin{array}{l}\text { S. anginosus } \\
\text { ATCC } 31412\end{array}$} & PLE & $1000 / 2000$ & $250 / 500$ & $0.25 / 0.25$ & \multirow{2}{*}{$0.75 / 0.75$} & \multirow{2}{*}{$\begin{array}{l}\text { Additive/ } \\
\text { Additive }\end{array}$} \\
\hline & Ampicillin & $0.063 / 0.25$ & $0.031 / 0.125$ & $0.5 / 0.5$ & & \\
\hline \multirow{2}{*}{$\begin{array}{l}\text { S. gordonii } \\
\text { ATCC } 10558\end{array}$} & PLE & $250 / 250$ & $63 / 125$ & $0.25 / 0.5$ & \multirow{2}{*}{$0.5 / 0.75$} & \multirow{2}{*}{$\begin{array}{l}\text { Synergistic/ } \\
\text { Additive }\end{array}$} \\
\hline & Ampicillin & $0.125 / 0.25$ & $0.031 / 0.063$ & $0.25 / 0.25$ & & \\
\hline \multirow{2}{*}{$\begin{array}{c}\text { A. actinomycetemcomitans } \\
\text { ATCC } 43717\end{array}$} & PLE & $2000 / 2000$ & $500 / 1000$ & $0.25 / 0.5$ & $05 / 10$ & Synergistic/ \\
\hline & Ampicillin & $16 / 32$ & $4 / 16$ & $0.25 / 0.5$ & $0.0 / 1.0$ & Additive \\
\hline F. nucleatum & PLE & $500 / 1000$ & $125 / 500$ & $0.25 / 0.5$ & 0 & Synergistic/ \\
\hline ATCC 51190 & Ampicillin & $4 / 16$ & $1 / 4$ & $0.25 / 0.25$ & $0.5 / 0.15$ & Additive \\
\hline
\end{tabular}




\begin{tabular}{|c|c|c|c|c|c|c|}
\hline \multirow{2}{*}{ Strains } & \multirow{2}{*}{ Agent } & \multicolumn{2}{|c|}{$\mathrm{MIC} / \mathrm{MBC}(\mu \mathrm{g} / \mathrm{mL})$} & \multirow{2}{*}{$\mathrm{FIC/FBC}$} & \multirow{2}{*}{$\begin{array}{l}\mathrm{FICI} / \\
\mathrm{FBCI}^{2}\end{array}$} & \multirow{2}{*}{ Outcome } \\
\hline & & Alone & Combination $^{1}$ & & & \\
\hline \multirow{2}{*}{$\begin{array}{l}\text { P. intermedia } \\
\text { ATCC } 49049\end{array}$} & PLE & $1000 / 2000$ & $250 / 250$ & $0.25 / 0.125$ & \multirow{2}{*}{$0.5 / 0.375$} & \multirow{2}{*}{$\begin{array}{l}\text { Synergistic/ } \\
\text { Synergistic }\end{array}$} \\
\hline & Ampicillin & $1 / 2$ & $0.25 / 0.5$ & $0.25 / 0.25$ & & \\
\hline \multirow{2}{*}{$\begin{array}{l}\text { P. gingivalis } \\
\text { ATCC } 33277\end{array}$} & PLE & $250 / 500$ & $63 / 125$ & $0.25 / 0.25$ & \multirow{2}{*}{$0.5 / 0.5$} & \multirow{2}{*}{$\begin{array}{l}\text { Synergistic } \\
\text { Synergistic }\end{array}$} \\
\hline & Ampicillin & $0.25 / 0.5$ & $0.063 / 0.125$ & $0.25 / 0.25$ & & \\
\hline
\end{tabular}

${ }^{1}$ The MIC and MBC of $50 \%$ ethanol extract of Paeonia lactiflora pallas (PLE) with ampicillin

${ }^{2}$ The fractional inhibitory concentration index (FIC index)

${ }^{3}$ American Type Culture Collection (ATCC)

${ }^{4}$ Korean collection for type cultures (KCTC)

Table 2: Synergistic effects of 50\% ethanol extract of Paeonia lactiflora pallas (PLE) with ampicillin against oral bacteria

\begin{tabular}{|c|c|c|c|c|c|c|}
\hline \multirow{2}{*}{ Strains } & \multirow{2}{*}{ Agent } & \multicolumn{2}{|c|}{$\mathrm{MIC} / \mathrm{MBC}(\mu \mathrm{g} / \mathrm{mL})$} & \multirow{2}{*}{ FIC } & \multirow{2}{*}{$\mathrm{FICI}^{2}$} & \multirow{2}{*}{ Outcome } \\
\hline & & Alone & Combination $^{1}$ & & & \\
\hline \multirow{2}{*}{$\begin{array}{l}\text { S. mutans } \\
\text { ATCC } 25175^{3}\end{array}$} & PLE & $250 / 250$ & $63 / 125$ & $0.25 / 0.5$ & \multirow{2}{*}{$0.75 / 0.75$} & \multirow{2}{*}{$\begin{array}{l}\text { Additive/ } \\
\text { Additive }\end{array}$} \\
\hline & Gentamicin & $8 / 16$ & $4 / 4$ & $0.5 / 0.25$ & & \\
\hline \multirow{2}{*}{$\begin{array}{l}\text { S. sanguinis } \\
\text { ATCC } 10556\end{array}$} & PLE & $500 / 1000$ & $125 / 500$ & $0.25 / 0.5$ & \multirow{2}{*}{$0.5 / 0.75$} & \multirow{2}{*}{$\begin{array}{c}\text { Synergistic/ } \\
\text { Additive }\end{array}$} \\
\hline & Gentamicin & $8 / 32$ & $2 / 8$ & $0.25 / 0.25$ & & \\
\hline \multirow{2}{*}{$\begin{array}{l}\text { S. parasanguinis } \\
\text { KCOM } 1497^{4}\end{array}$} & PLE & $1000 / 1000$ & $250 / 500$ & $0.25 / 0.5$ & \multirow{2}{*}{$0.5 / 0.75$} & \multirow{2}{*}{$\begin{array}{c}\text { Synergistic/ } \\
\text { Additive }\end{array}$} \\
\hline & Gentamicin & $16 / 32$ & $4 / 8$ & $0.25 / 0.25$ & & \\
\hline \multirow{2}{*}{$\begin{array}{c}\text { S. sobrinus } \\
\text { ATCC } 27607\end{array}$} & PLE & $500 / 1000$ & $125 / 250$ & $0.25 / 0.25$ & \multirow{2}{*}{$0.5 / 0.5$} & \multirow{2}{*}{$\begin{array}{c}\text { Synergistic/ } \\
\text { Synergistic }\end{array}$} \\
\hline & Gentamicin & $8 / 32$ & $2 / 8$ & $0.25 / 0.25$ & & \\
\hline \multirow{2}{*}{$\begin{array}{c}\text { S. ratti } \\
\text { KCTC } 3294^{5}\end{array}$} & PLE & $500 / 1000$ & $125 / 500$ & $0.25 / 0.5$ & \multirow{2}{*}{$0.5 / 1.0$} & \multirow{2}{*}{$\begin{array}{l}\text { Synergistic/ } \\
\text { Additive }\end{array}$} \\
\hline & Gentamicin & $8 / 16$ & $2 / 8$ & $0.25 / 0.5$ & & \\
\hline \multirow{2}{*}{$\begin{array}{c}\text { S. criceti } \\
\text { KCTC } 3292\end{array}$} & PLE & $500 / 2000$ & $125 / 500$ & $0.25 / 0.25$ & \multirow{2}{*}{$0.5 / 0.5$} & \multirow{2}{*}{$\begin{array}{c}\text { Synergistic/ } \\
\text { Synergistic }\end{array}$} \\
\hline & Gentamicin & $16 / 32$ & $4 / 8$ & $0.25 / 0.25$ & & \\
\hline \multirow{2}{*}{$\begin{array}{l}\text { S. downei } \\
\text { KCOM } 1165\end{array}$} & PLE & $1000 / 1000$ & $500 / 500$ & $0.5 / 0.5$ & \multirow{2}{*}{$0.75 / 0.75$} & \multirow{2}{*}{$\begin{array}{l}\text { Additive/ } \\
\text { Additive }\end{array}$} \\
\hline & Gentamicin & $32 / 64$ & $8 / 16$ & $0.25 / 0.25$ & & \\
\hline \multirow{2}{*}{$\begin{array}{l}\text { S. anginosus } \\
\text { ATCC } 31412\end{array}$} & PLE & $1000 / 2000$ & $250 / 500$ & $0.25 / 0.25$ & \multirow{2}{*}{$0.5 / 0.5$} & \multirow{2}{*}{$\begin{array}{l}\text { Synergistic/ } \\
\text { Synergistic }\end{array}$} \\
\hline & Gentamicin & $8 / 16$ & $2 / 4$ & $0.25 / 0.25$ & & \\
\hline \multirow{2}{*}{$\begin{array}{l}\text { S. gordonii } \\
\text { ATCC } 10558\end{array}$} & PLE & $250 / 250$ & $63 / 125$ & $0.25 / 0.5$ & \multirow{2}{*}{$0.5 / 0.75$} & Synergistic/ \\
\hline & Gentamicin & $16 / 32$ & $4 / 8$ & $0.25 / 0.25$ & & Additive \\
\hline A. actinomycetemcomitans & PLE & $2000 / 2000$ & $500 / 1000$ & $0.25 / 0.5$ & 103751075 & Synergistic/ \\
\hline ATCC 43717 & Gentamicin & $16 / 16$ & $2 / 4$ & $0.125 / 0.25$ & $0.2 / 5 / 0.15$ & Additive \\
\hline F. nucleatum & PLE & $500 / 1000$ & $125 / 250$ & $0.25 / 0.25$ & $0.5 / 0.5$ & Synergistic/ \\
\hline ATCC 51190 & Gentamicin & $2 / 4$ & $0.5 / 1$ & $0.25 / 0.25$ & & Synergistic \\
\hline P. intermedia & PLE & $1000 / 2000$ & $250 / 500$ & $0.25 / 0.25$ & 05105 & Synergistic/ \\
\hline ATCC 25611 & Gentamicin & $32 / 64$ & $8 / 16$ & $0.25 / 0.25$ & $0.3 / 0.3$ & Synergistic \\
\hline P. gingivalis & PLE & $250 / 500$ & $63 / 125$ & $0.25 / 0.25$ & $05 / 05$ & Synergistic/ \\
\hline ATCC 33277 & Gentamicin & $256 / 512$ & $64 / 128$ & $0.25 / 0.25$ & $0.3 / 0.3$ & Synergistic \\
\hline
\end{tabular}

${ }^{1}$ The MIC and MBC of $50 \%$ ethanol extract of Paeonia lactiflora pallas (PLE) with gentamicin

${ }^{2}$ The fractional inhibitory concentration index (FIC index)

${ }^{3}$ American Type Culture Collection (ATCC)

${ }^{4}$ Korean collection for type cultures (KCTC)

Table 3: Synergistic effects of 50\% ethanol extract of Paeonia lactiflora pallas (PLE) with gentamicin against oral bacteria

\begin{tabular}{|c|c|c|c|c|c|c|}
\hline \multirow{2}{*}{ Strains } & \multirow{2}{*}{ Agent } & \multicolumn{2}{|c|}{ MIC/MBC $(\mu \mathrm{g} / \mathrm{mL})$} & \multirow{2}{*}{ FIC } & \multirow{2}{*}{$\mathbf{F I C I}^{2}$} & \multirow{2}{*}{ Outcome } \\
\hline & & Alone & Combination $^{1}$ & & & \\
\hline \multirow{2}{*}{$\begin{array}{l}\text { S. mutans } \\
\text { ATCC } 25175^{3}\end{array}$} & PLE & $250 / 250$ & $63 / 125$ & $0.25 / 0.5$ & \multirow{2}{*}{$0.5 / 1.0$} & \multirow{2}{*}{$\begin{array}{c}\text { Synergistic/ } \\
\text { Additive }\end{array}$} \\
\hline & Erythromycin & $0.063 / 0.125$ & $0.016 / 0.063$ & $0.25 / 0.5$ & & \\
\hline \multirow{2}{*}{$\begin{array}{l}\text { S. sanguinis } \\
\text { ATCC } 10556\end{array}$} & PLE & $500 / 1000$ & $125 / 250$ & $0.25 / 0.25$ & \multirow{2}{*}{$0.75 / 0.75$} & \multirow{2}{*}{$\begin{array}{l}\text { Additive/ } \\
\text { Additive }\end{array}$} \\
\hline & Erythromycin & $0.016 / 0.031$ & $0.007 / 0.016$ & $0.5 / 0.5$ & & \\
\hline \multirow{2}{*}{$\begin{array}{l}\text { S. parasanguinis } \\
\text { KCOM } 1497^{4}\end{array}$} & PLE & $1000 / 1000$ & $250 / 250$ & $0.25 / 0.25$ & \multirow{2}{*}{$0.5 / 0.5$} & \multirow{2}{*}{$\begin{array}{l}\text { Synergistic/ } \\
\text { Synergistic }\end{array}$} \\
\hline & Erythromycin & $0.25 / 0.5$ & $0.063 / 0.125$ & $0.25 / 0.25$ & & \\
\hline
\end{tabular}




\begin{tabular}{|c|c|c|c|c|c|c|}
\hline \multirow{2}{*}{ Strains } & \multirow{2}{*}{ Agent } & \multicolumn{2}{|c|}{ MIC/MBC $(\mu \mathrm{g} / \mathrm{mL})$} & \multirow{2}{*}{ FIC } & \multirow{2}{*}{$\mathrm{FICI}^{2}$} & \multirow{2}{*}{ Outcome } \\
\hline & & Alone & Combination $^{1}$ & & & \\
\hline \multirow{2}{*}{$\begin{array}{l}\text { S. sobrinus } \\
\text { ATCC } 27607\end{array}$} & PLE & $500 / 1000$ & $250 / 500$ & $0.5 / 0.5$ & \multirow{2}{*}{$1.0 / 0.75$} & \multirow{2}{*}{$\begin{array}{l}\text { Additive/ } \\
\text { Additive }\end{array}$} \\
\hline & Erythromycin & $0.031 / 0.063$ & $0.016 / 0.031$ & $0.5 / 0.25$ & & \\
\hline \multirow{2}{*}{$\begin{array}{c}\text { S. ratti } \\
\text { KCTC } 3294^{5}\end{array}$} & PLE & $500 / 1000$ & $125 / 250$ & $0.25 / 0.25$ & \multirow{2}{*}{$0.5 / 0.5$} & \multirow{2}{*}{$\begin{array}{l}\text { Synergistic/ } \\
\text { Synergistic }\end{array}$} \\
\hline & Erythromycin & $0.008 / 0.016$ & $0.002 / 0.004$ & $0.25 / 0.25$ & & \\
\hline \multirow{2}{*}{$\begin{array}{c}\text { S. criceti } \\
\text { KCTC } 3292\end{array}$} & PLE & $500 / 2000$ & $125 / 250$ & $0.25 / 0.125$ & \multirow{2}{*}{$0.5 / 0.375$} & \multirow{2}{*}{$\begin{array}{c}\text { Synergistic/ } \\
\text { Synergistic }\end{array}$} \\
\hline & Erythromycin & $0.125 / 0.25$ & $0.031 / 0.063$ & $0.25 / 0.25$ & & \\
\hline \multirow{2}{*}{$\begin{array}{l}\text { S. downei } \\
\text { KCOM } 1165\end{array}$} & PLE & $1000 / 1000$ & $125 / 250$ & $0.125 / 0.25$ & \multirow{2}{*}{$0.375 / 0.5$} & \multirow{2}{*}{$\begin{array}{l}\text { Synergistic/ } \\
\text { Synergistic }\end{array}$} \\
\hline & Erythromycin & $0.25 / 0.5$ & $0.063 / 0.125$ & $0.25 / 0.25$ & & \\
\hline \multirow{2}{*}{$\begin{array}{l}\text { S. anginosus } \\
\text { ATCC } 31412\end{array}$} & PLE & $1000 / 2000$ & $250 / 250$ & $0.25 / 0.125$ & \multirow{2}{*}{$0.5 / 0.375$} & \multirow{2}{*}{$\begin{array}{l}\text { Synergistic/ } \\
\text { Synergistic }\end{array}$} \\
\hline & Erythromycin & $0.25 / 0.5$ & $0.063 / 0.125$ & $0.25 / 0.25$ & & \\
\hline \multirow{2}{*}{$\begin{array}{l}\text { S. gordonii } \\
\text { ATCC } 10558\end{array}$} & PLE & $250 / 250$ & $63 / 125$ & $0.25 / 0.5$ & \multirow{2}{*}{$0.5 / 0.75$} & \multirow{2}{*}{$\begin{array}{c}\text { Synergistic/ } \\
\text { Additive }\end{array}$} \\
\hline & Erythromycin & $0.031 / 0.063$ & $0.008 / 0.016$ & $0.25 / 0.25$ & & \\
\hline \multirow{2}{*}{$\begin{array}{l}\text { A. actinomycetemcomitans } \\
\text { ATCC } 43717\end{array}$} & PLE & $2000 / 2000$ & $500 / 500$ & $0.25 / 0.25$ & \multirow{2}{*}{$0.375 / 0.5$} & \multirow{2}{*}{$\begin{array}{l}\text { Synergistic/ } \\
\text { Synergistic }\end{array}$} \\
\hline & Erythromycin & $0.125 / 0.25$ & $0.016 / 0.031$ & $0.125 / 0.25$ & & \\
\hline \multirow{2}{*}{$\begin{array}{l}\text { F. nucleatum } \\
\text { ATCC } 51190\end{array}$} & PLE & $500 / 1000$ & $125 / 250$ & $0.25 / 0.25$ & \multirow{2}{*}{$0.5 / 0.5$} & \multirow{2}{*}{$\begin{array}{l}\text { Synergistic/ } \\
\text { Synergistic }\end{array}$} \\
\hline & Erythromycin & $32 / 64$ & $8 / 16$ & $0.25 / 0.25$ & & \\
\hline \multirow{2}{*}{$\begin{array}{l}\text { P. intermedia } \\
\text { ATCC } 25611\end{array}$} & PLE & $1000 / 2000$ & $250 / 500$ & $0.25 / 0.25$ & \multirow{2}{*}{$0.5 / 0.5$} & \multirow{2}{*}{$\begin{array}{l}\text { Synergistic/ } \\
\text { Synergistic }\end{array}$} \\
\hline & Erythromycin & $16 / 32$ & $4 / 8$ & $0.25 / 0.25$ & & \\
\hline \multirow{2}{*}{$\begin{array}{c}\text { P. gingivalis } \\
\text { ATCC } 33277\end{array}$} & PLE & $250 / 500$ & $63 / 63$ & $0.25 / 0.125$ & \multirow{2}{*}{$0.75 / 0.375$} & Additive/ \\
\hline & Erythromycin & $2 / 8$ & $1 / 2$ & $0.5 / 0.25$ & & Synergistic \\
\hline
\end{tabular}

${ }^{1}$ The MIC and MBC of 50\% ethanol extract of Paeonia lactiflora pallas (PLE) with erythromycin

${ }^{2}$ The fractional inhibitory concentration index (FIC index)

${ }^{3}$ merican Type Culture Collection (ATCC)

${ }^{4}$ Korean collection for type cultures (KCTC)

Table 4: Synergistic effects of 50\% ethanol extract of Paeonia lactiflora pallas (PLE) with erythromycin against oral bacteria

\begin{tabular}{|c|c|c|c|c|c|c|}
\hline \multirow{2}{*}{ Strains } & \multirow{2}{*}{ Agent } & \multicolumn{2}{|c|}{ MIC/MBC $(\mu \mathrm{g} / \mathrm{mL})$} & \multirow{2}{*}{ FIC } & \multirow{2}{*}{$\mathrm{FICI}^{2}$} & \multirow{2}{*}{ Outcome } \\
\hline & & Alone & Combination $^{1}$ & & & \\
\hline \multirow{2}{*}{$\begin{array}{l}\text { S. mutans } \\
\text { ATCC } 25175^{3}\end{array}$} & PLE & $250 / 250$ & $63 / 63$ & $0.25 / 0.25$ & \multirow{2}{*}{$0.5 / 0.5$} & \multirow{2}{*}{$\begin{array}{l}\text { Synergistic/ } \\
\text { Synergistic }\end{array}$} \\
\hline & Vancomycin & $1 / 2$ & $0.25 / 0.5$ & $0.25 / 0.25$ & & \\
\hline \multirow{2}{*}{$\begin{array}{l}\text { S. sanguinis } \\
\text { ATCC } 10556\end{array}$} & PLE & $500 / 1000$ & $125 / 250$ & $0.25 / 0.25$ & \multirow{2}{*}{$0.5 / 0.75$} & \multirow{2}{*}{$\begin{array}{l}\text { Synergistic/ } \\
\text { Additive }\end{array}$} \\
\hline & Vancomycin & $0.5 / 1$ & $0.125 / 0.5$ & $0.25 / 0.5$ & & \\
\hline \multirow{2}{*}{$\begin{array}{l}\text { S. parasanguinis } \\
\text { KCOM } 1497^{4}\end{array}$} & PLE & $1000 / 1000$ & $250 / 250$ & $0.25 / 0.25$ & \multirow{2}{*}{$0.75 / 0.75$} & \multirow{2}{*}{$\begin{array}{l}\text { Additive/ } \\
\text { Additive }\end{array}$} \\
\hline & Vancomycin & $2 / 4$ & $1 / 2$ & $0.5 / 0.5$ & & \\
\hline \multirow{2}{*}{$\begin{array}{l}\text { S. sobrinus } \\
\text { ATCC } 27607\end{array}$} & PLE & $500 / 1000$ & $125 / 250$ & $0.25 / 0.25$ & \multirow{2}{*}{$0.75 / 0.5$} & \multirow{2}{*}{$\begin{array}{l}\text { Additive/ } \\
\text { Synergistic }\end{array}$} \\
\hline & Vancomycin & $1 / 2$ & $0.5 / 0.5$ & $0.5 / 0.25$ & & \\
\hline \multirow{2}{*}{$\begin{array}{l}\text { S. ratti } \\
\text { KCTC } 3294^{5}\end{array}$} & PLE & $500 / 1000$ & $125 / 250$ & $0.25 / 0.25$ & \multirow{2}{*}{$0.5 / 0.5$} & \multirow{2}{*}{$\begin{array}{l}\text { Synergistic/ } \\
\text { Synergistic }\end{array}$} \\
\hline & Vancomycin & $1 / 1$ & $0.25 / 0.25$ & $0.25 / 0.25$ & & \\
\hline \multirow{2}{*}{$\begin{array}{c}\text { S. criceti } \\
\text { KCTC } 3292\end{array}$} & PLE & $500 / 2000$ & $125 / 500$ & $0.25 / 0.25$ & \multirow{2}{*}{$0.75 / 0.5$} & \multirow{2}{*}{$\begin{array}{l}\text { Additive/ } \\
\text { Synergistic }\end{array}$} \\
\hline & Vancomycin & $2 / 4$ & $1 / 1$ & $0.5 / 0.25$ & & \\
\hline \multirow{2}{*}{$\begin{array}{l}\text { S. downei } \\
\text { KCOM } 1165\end{array}$} & PLE & $1000 / 1000$ & $250 / 250$ & $0.25 / 0.25$ & \multirow{2}{*}{$0.5 / 0.5$} & \multirow{2}{*}{$\begin{array}{l}\text { Additive/ } \\
\text { Synergistic }\end{array}$} \\
\hline & Vancomycin & $8 / 16$ & $2 / 4$ & $0.25 / 0.25$ & & \\
\hline \multirow{2}{*}{$\begin{array}{l}\text { S. anginosus } \\
\text { ATCC } 31412\end{array}$} & PLE & $1000 / 2000$ & $250 / 500$ & $0.25 / 0.25$ & \multirow{2}{*}{$0.5 / 0.375$} & \multirow{2}{*}{$\begin{array}{l}\text { Synergistic/ } \\
\text { Synergistic }\end{array}$} \\
\hline & Vancomycin & $1 / 4$ & $0.25 / 0.5$ & $0.25 / 0.125$ & & \\
\hline \multirow{2}{*}{$\begin{array}{l}\text { S. gordonii } \\
\text { ATCC } 10558\end{array}$} & PLE & $250 / 250$ & $63 / 125$ & $0.25 / 0.5$ & \multirow{2}{*}{$0.5 / 0.75$} & \multirow{2}{*}{$\begin{array}{l}\text { Synergistic/ } \\
\text { Additive }\end{array}$} \\
\hline & Vancomycin & $0.5 / 1$ & $0.125 / 0.25$ & $0.25 / 0.25$ & & \\
\hline \multirow{2}{*}{$\begin{array}{l}\text { A. actinomycetemcomitans } \\
\text { ATCC } 43717\end{array}$} & PLE & $2000 / 2000$ & $500 / 1000$ & $0.25 / 0.5$ & $0.5 / 075$ & Synergistic/ \\
\hline & Vancomycin & $2 / 4$ & $0.5 / 1$ & $0.25 / 0.25$ & $0.5 / 0.15$ & Additive \\
\hline F. nucleatum & PLE & $500 / 1000$ & $125 / 250$ & $0.25 / 0.25$ & 10.5 & Synergistic/ \\
\hline ATCC 51190 & Vancomycin & $64 / 128$ & $8 / 32$ & $0.125 / 0.25$ & $0.3 / 5 / 0.3$ & Synergistic \\
\hline
\end{tabular}




\begin{tabular}{|c|c|c|c|c|c|c|}
\hline \multirow{2}{*}{ Strains } & \multirow{2}{*}{ Agent } & \multicolumn{2}{|c|}{ MIC/MBC $(\mu \mathrm{g} / \mathrm{mL})$} & \multirow{2}{*}{ FIC } & \multirow{2}{*}{ FICI $^{2}$} & \multirow{2}{*}{ Outcome } \\
\hline & & Alone & Combination $^{1}$ & & & \\
\hline \multirow{2}{*}{$\begin{array}{l}\text { P. intermedia } \\
\text { ATCC } 25611\end{array}$} & PLE & $1000 / 2000$ & $250 / 250$ & $0.25 / 0.125$ & \multirow{2}{*}{$0.5 / 0.375$} & \multirow{2}{*}{$\begin{array}{l}\text { Synergistic/ } \\
\text { Synergistic }\end{array}$} \\
\hline & Vancomycin & $16 / 36$ & $4 / 8$ & $0.25 / 0.25$ & & \\
\hline \multirow{2}{*}{$\begin{array}{l}\text { P. gingivalis } \\
\text { ATCC } 33277\end{array}$} & PLE & $250 / 500$ & $63 / 125$ & $0.25 / 0.25$ & \multirow{2}{*}{$0.375 / 0.5$} & \multirow{2}{*}{$\begin{array}{l}\text { Synergistic/ } \\
\text { Synergistic }\end{array}$} \\
\hline & Vancomycin & $16 / 16$ & $2 / 4$ & $0.125 / 0.25$ & & \\
\hline
\end{tabular}

${ }^{1}$ The MIC and MBC of $50 \%$ ethanol extract of Paeonia lactiflora pallas (PLE) with vancomycin

${ }^{2}$ The fractional inhibitory concentration index (FIC index)

${ }^{3}$ American Type Culture Collection (ATCC)

${ }^{4}$ Korean collection for type cultures (KCTC)

Table 5: Synergistic effects of 50\% ethanol extract of Paeonia lactiflora pallas (PLE) with vancomycin against oral bacteria

The major bioactive components of $P$. lactiflora include monoterpene glycosides, lignans, galloyl glucoses, nortriterpenoids, and phenolic compounds $[16,17,22]$. The extract of $P$. lactiflora has been demonstrated to exhibit anti-inflammatory, anti-spasmodic, and antiviral activities, in addition to lowering uric acid concentrations [14,15,22,23]. Recently, methyl gallate, paeonol, and 1,2,3,4,6-penta-O-galloyl- $\beta$-d-glucopyranose (PGG) with $P$. lactiflora root extract have been suggested as alternative sources of anti-Helicobacter pylori products because of their growth-inhibiting, bactericidal, and urease inhibitory effects [23]. Furthermore, it has been described that phenolic acids can break down the structure of the cytoplasmic membrane causing loss of integrity and eventual cell death [24]. At sub-inhibitory concentrations, the compounds present in the extract would facilitate the entrance of the antibiotic to the cell cytoplasm, thus facilitating the entrance of ampicillin, gentamicin, erythromycin, and vancomycin, which have their site action within the bacterial cell, and less antibiotic dose would be needed. In this way, the multi-objective mechanism would be accomplished by disrupting the cytoplasmic membrane and some vital function as DNA replication, transcription or translation processes, depending on the antibiotic used [24,25].

The bacterial effect of the PLE with ampicillin, gentamicin, erythromycin, and/or vancomycin against oral bacteria was confirmed by time-kill curve experiments. The PLE (MIC or 1/2 MIC) alone resulted rate of killing increasing or not changing in CFU/ml at time-dependent manner, with a more rapid rate of killing by ARE (1/2 MIC) with ampicillin (1/2 MIC), gentamicin (1/2 MIC), erythromycin (1/2 MIC), and/or vancomycin (1/2 MIC) (Figure 1 and 2). The PLE with ampicillin (1/2 MIC) and vancomycin combination was bactericidal effect up to and beyond $12 \mathrm{~h}$ exposure with all killing compared to PLE alone in all tested bacteria. The PLE with gentamicin (1/2 MIC) combination was bactericidal effect with all killing up to $12 \mathrm{~h}$ exposure in all tested bacteria except $S$. sanguinis, S. sobrinus, and $S$. ratti, and with erythromycin except $S$. sobrinus and $S$. ratti.
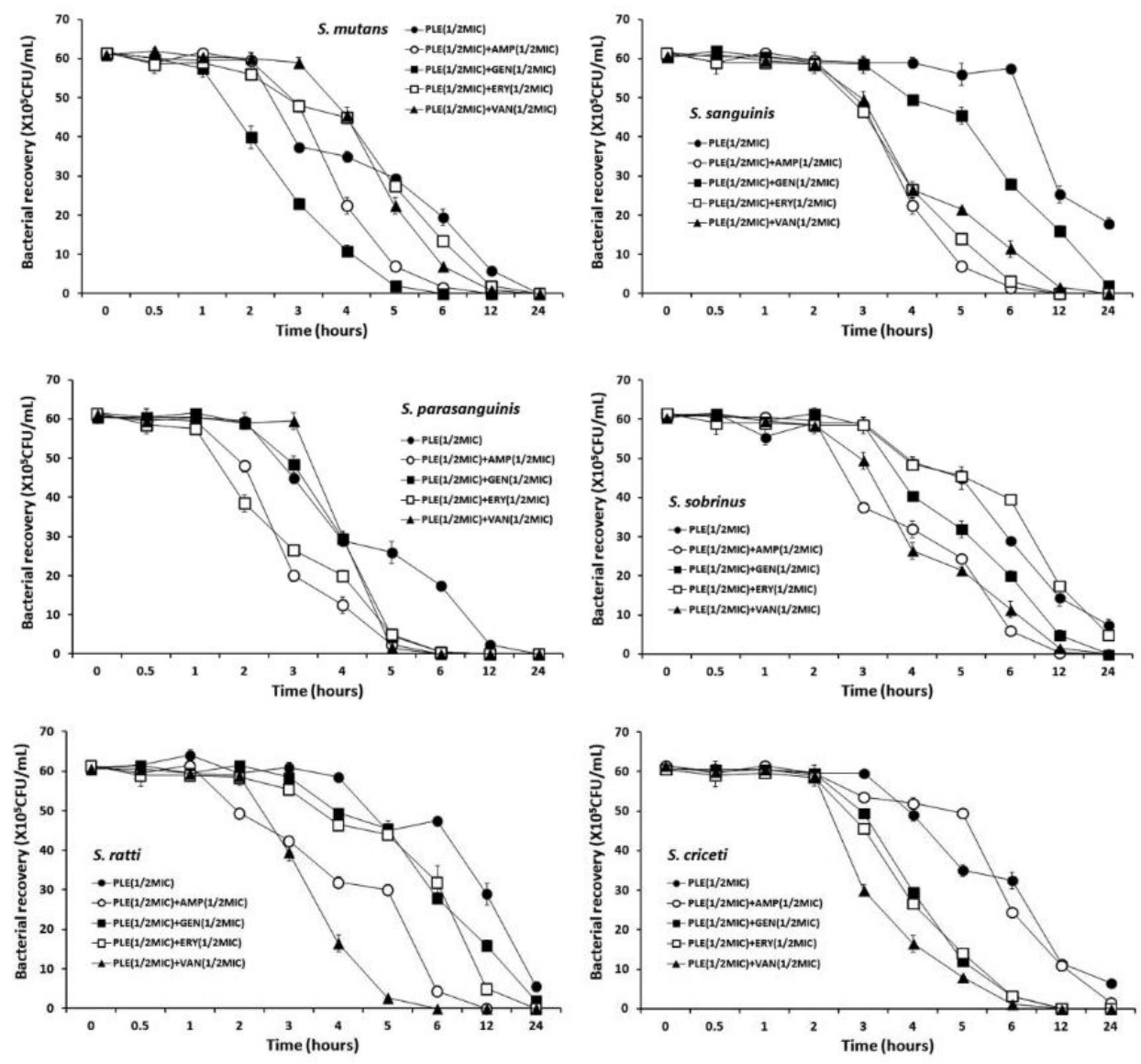

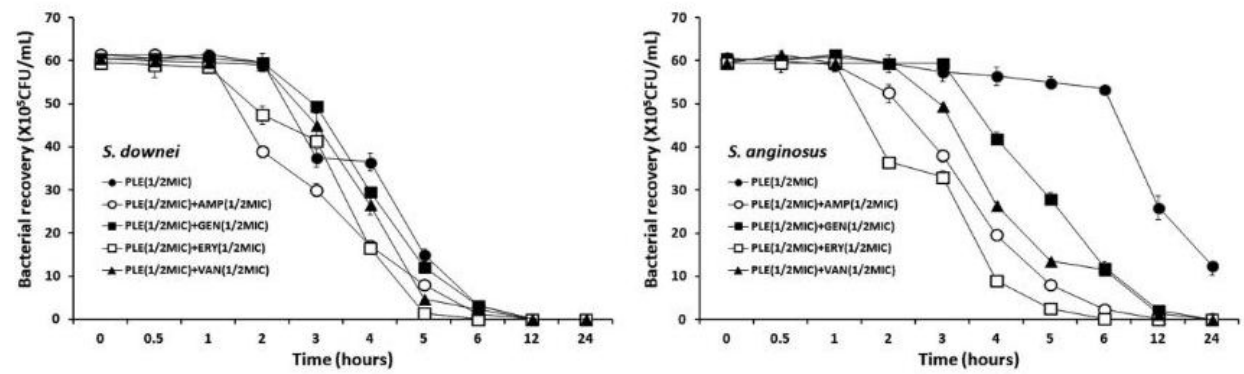

Figure 1: Time-kill curves of MIC of PLE alone and its combination with 1/2 MIC of AMP, GEN, ERY, or/and VAN against $S$. mutans, S. sanguinis, S. parasanguinis, S. sobrinus, S. ratti, S. criceti, S. downei, and S. anginosus. Bacteria were incubated with $\operatorname{PLE}(\bullet), \operatorname{PLE}+\operatorname{AMP}(\mathrm{O}), \operatorname{PLE}+\operatorname{GEN}(\boldsymbol{\Delta}), \operatorname{PLE}+\operatorname{ERY}(\Delta)$, and PLE + VAN $(\bullet)$ over time. Data points are the mean values \pm S.E.M. of six experiments. CFU, colony-forming units
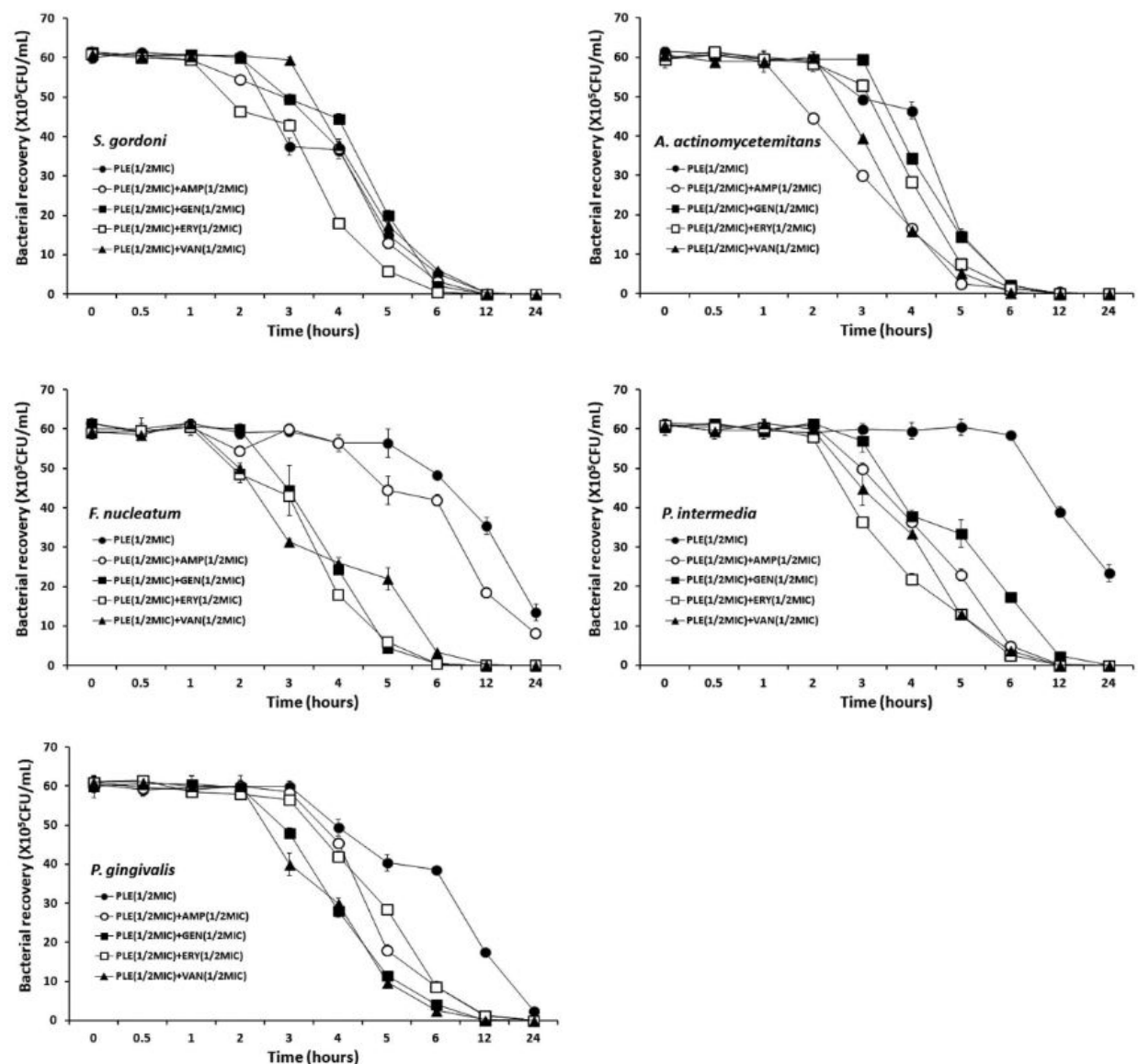

Figure 2: Time-kill curves of MIC of PLE alone and its combination with 1/2 MIC of AMP, GEN, ERY, or/and VAN against $S$. gordonii, A. actinomycetemcomitans, F. nucleatum, P. intermedia, and P. gingivalis. Bacteria were incubated with PLE $(\bullet), \operatorname{PLE}+\operatorname{AMP}(O), \operatorname{PLE}+\operatorname{GEN}(\boldsymbol{\Lambda}), \operatorname{PLE}+\operatorname{ERY}(\Delta)$, and PLE + VAN (•) over time. Data points are the mean values \pm S.E.M. of six experiments. CFU, colony-forming units

\section{Conclusion}

In conclusion, these findings suggest that a strong bactericidal effect of PLE was exerted in drug combinations and fulfills the conditions required of a novel cariogenic bacteria and periodontal pathogens, particularly bacteroides species drug and may be useful in the future in the treatment of oral bacteria.

\section{Competing Interest}

The authors declare that they have no competing interests.

\section{Acknowledgment}

This research was supported by Basic Science Research Program through the National Research Foundation of Korea (NRF) funded by the Ministry of Education, Science and Technology (KRF-20110023479). 


\section{References}

1. Patil S, Rao RS, Sanketh DS, Amrutha N (2013) Microbial flora in oral diseases. J Contemp Dent Pract 14: 1202-8.

2. López R, Smith PC, Göstemeyer G, Schwendicke F (2017) Ageing, dental caries and periodontal diseases. J Clin Periodontol 44 Suppl 18: S145-S152.

3. Chi AC, Neville BW, Krayer JW, Gonsalves WC (2010) Oral manifestations of systemic disease. Am Fam Physician 82:1381-8.

4. Pitts NB, Zero DT, Marsh PD, Ekstrand K, Weintraub JA, et al. (2017) Dental caries. Nat Rev Dis Primers 3: 17030.

5. Cardoso EM, Reis C, Manzanares-Céspedes MC (2018) Chronic periodontitis, inflammatory cytokines, and interrelationship with other chronic diseases. Postgrad Med 130: 98-104.

6. Kinane DF, Stathopoulou PG, Papapanou PN (2017) Periodontal diseases. Nat Rev Dis Primers 3: 17038.

7. Aoyama N, Suzuki JI, Kobayashi N, Hanatani T, Ashigaki N, et al. (2018) Associations among tooth loss, systemic inflammation and antibody titers to periodontal pathogens in Japanese patients with cardiovascular disease. J Periodontal Res 53: 117-122.

8. Rowson C, Townsend R (2016) Biofilms: prevention and treatment. Br J Hosp Med 77: 699-703.

9. Levi ME, Eusterman VD (2011) Oral infections and antibiotic therapy. Otolaryngol Clin North Am 44: 57-78.

10. Rams TE, Degener JE, van Winkelhoff AJ (2013) Prevalence of beta-lactamase-producing bacteria in human periodontitis. J Periodontal Res 48: 493-9.

11. Kocsis B, Domokos J, Szabo D (2016) Chemical structure and pharmacokinetics of novel quinolone agents represented by avarofloxacin, delafloxacin, finafloxacin, zabofloxacin and nenonoxacin. Ann Clin Microbiol Antimicrob 15: 34.

12. Mishra BB, Tiwari VK (2011) Natural products: an evolving role in future drug discovery. Eur J Med Chem 46: 4769-807.

13. Zhang W, Dai SM (2012) Mechanisms involved in the therapeutic effects of Paeonia lactiflora Pallas in rheumatoid arthritis. Int Immunopharmacol 14: 27-31.

14. Jiang D, Chen Y, Hou X, Xu J, Mu X, et al. (2011) Influence of Paeonia lactiflora roots extract on cAMP-phosphodiesterase activity and related anti-inflammatory action. J Ethnopharmacol 137: 914-20.

15. Ho JY, Chang HW, Lin CF, Liu CJ, Hsieh CF, et al. (2014) Characterization of the anti-influenza activity of the Chinese herbal plant Paeonia lactiflora. Viruses 6: 1861-75.

16. Li YC, Wang LY, Li R, Wu YZ, Guo QL, et al. (2016) Chemical constituents from ethyl actate exaction of root of Paeonia lactiflora. Zhongguo Zhong Yao Za Zhi 41: 2255-60.

17. Kim JS, Kim YJ, Lee JY, Kang SS (2008) Phytochemical studies on Paeoniae Radix (2)-Phenolic and related compounds. Kor J Pharmacogn 39: 28-36.

18. Abdel-Hafez AA, Meselhy MR, Nakamura N, Hattori M, Watanabe H, et al. (1999) Anticonvulsant activity of paeonimetabolin-I adducts obtained by incubation of paeoniflorin and thiol compounds with Lactobacillus brevis. Biol Pharm Bull 22: 491-7.

19. Adwan G, Abu-Shanab B, Adwan K (2010) Antibacterial activities of some plant extracts alone and in combination with different antimicrobials against multidrug-resistant Pseudomonas aeruginosa strains. Asian Pac J Trop Biomed 1: 266-9.

20. Aiyegoro OA, Okoh AI (2009) Use of bioactive plant products in combination with standard antibiotics: Implications in antimicrobial chemotherapy. J Med Plant Res 3: 1147-52.

21. Sanhueza L, Melo R, Montero R, Maisey K, Mendoza L, et al. (2017) Synersistic interactions between phenolic compounds identified in grape pomace extract with antibiotics of different classes against Staphylococcus aureus and Escherichia coli. PLoS One 12: e0172273.

22. Fu Q, Qiu L, Yuan H. Yu M, Zou T, et al. (2016) Paeonenoides D and E: two new nortriterpenoids from Paeonia lactiflora and their inhibitory activities on NO production. Helv Chim Acta 99: 46-9.

23. Ngan LT, Moon JK, Shibamoto T, Ahn YJ (2012) Growth-inhibiting, bactericidal, and urease inhibitory effects of Paeonia lactiflora root constituents and related compounds on antibiotic-susceptible and -resistant strains of Helicobacter pylori. J Agric Food Chem 60: 9062-73.

24. Cowan MM (1999) Plant products as antimicrobial agents. Clin Microbiol Rev 12: 564-82.

25. Xie Y, Yang W, Tang F, Chen X, Ren L (2015) Antibacterial activities of flavonoids: structure-activity relationship and mechanism. Curr Med Chem 22: 132-49. 